\section{Absence of opiate rewarding effects in mice lacking dopamine D2 receptors}

\section{Rafael Maldonado ${ }^{*}$, Adolfo Saiardi $\dagger$, Olga Valverde*, Tarek A. Samad $\dagger$, Bernard P. Roques ${ }^{\star}$ \& Emiliana Borrelli $\dagger$}

* Département de Pharmacochimie Moléculaire et Structurale, INSERM U266, URA D1500 CNRS, Université René Descartes, 75270 Paris, France

$\dagger$ Institut de Génétique et de Biologie Moléculaire et Cellulaire, INSERM/CNRS/ ULP, BP 163, 67404 Illkirch, C.U. de Strasbourg, France

Dopamine receptors have been implicated in the behavioural response to drugs of abuse. These responses are mediated particularly by the mesolimbic dopaminergic pathway arising in the ventral tegmental area and projecting to the limbic system. The rewarding properties of opiates ${ }^{1}$ and the somatic expression of morphine abstinence ${ }^{2}$ have been related to changes in mesolimbic dopaminergic activity that could constitute the neural substrate for opioid addiction ${ }^{3}$. These adaptive responses to repeated morphine administration have been investigated in mice with a genetic disruption of the dopaminergic D2 receptors ${ }^{4}$. Although the behavioural expression of morphine withdrawal was unchanged in these mice, a total suppression of morphine rewarding properties was observed in a place-preference test. This effect is specific to the drug, as mice lacking D2 receptors behaved the same as wild-type mice when food is used as reward. We conclude that the D2 receptor plays a crucial role in the motivational component of drug addiction.

Dopamine released by dopaminergic neurons in the ventral tegmental area exerts a negative control on the mesolimbic dopaminergic pathway by activating somato-dendritic D2 autoreceptors ${ }^{3,5}$. The firing of these neurons is negatively regulated by GABA-mediated interneurons. This control is potentiated by stimulation of GABA receptors and is reduced by the inhibition of GABA release ${ }^{6}$. Opioids indirectly relieve this negative control by inhibiting the firing of these neurons. This mechanism has been proposed to mediate the rewarding effects of morphine and heroin ${ }^{3}$. Indeed, opiate injections into this area generate selfadministration $^{7}$, potentiate rewarding brain stimulation ${ }^{8}$, and produce a conditioned place preference ${ }^{9}$. These behaviours seem to depend on an increased dopaminergic activity ${ }^{10,11}$, although a dopamine-independent mechanism has also been postulated ${ }^{12-14}$. Moreover, the participation of the dopaminergic mesolimbic system in physical opiate dependence has also been reported ${ }^{2,15,16}$. Particularly, D2 receptors (D2Rs) within the nucleus accumbens have been shown to be involved in the expression of the somatic symptoms of opiate withdrawal' ${ }^{2}$.

To establish the role of dopamine D2R in such functions, we analysed the behavioural response of D2R-null mice ${ }^{4}$ to morphine. We have previously shown that D2R-null mice present a decrease in spontaneous locomotor activity ${ }^{4}$. However, D2R-deficient mice compared with their wild-type littermates ( $n=12$ per group) retain exploratory behaviour in a stressful environment (Fig. 1a). A normal habituation to the environment after three consecutive sessions is also observed (Fig. 1a). To investigate whether morphine would induce hyperlocomotion in D2R-null mice, as it does in wildtype mice ${ }^{17}$, animals were tested in locomotor-activity boxes under non-stressful conditions. Morphine administration at doses of 2 and $6 \mathrm{mg}$ per $\mathrm{kg}$ body weight ( $n=6$ per group) induced a similar increase in horizontal locomotor activity in both wild-type and D2R-null mice (Fig. 1b).

The administration of RB 101 (100 mg per $\mathrm{kg}$ body weight, intraperitoneal (i.p.)), a mixed inhibitor of enkephalin-degrading enzymes $^{18}$, also induced hyperactivity in both groups. Indeed, a significant increase $(P<0.01)$ in horizontal locomotor activity was observed in RB101-treated wild-type animals (32 $\pm 7 \%)$ and D2Rnull mice $(48 \pm 15 \%)$ in comparison to saline-treated animals (Student's $t$-test: $t_{(1,12)}=3.613, P<0.01$, and $t_{(1,13)}=2.505$, $P<0.05$, respectively). Consequently, the motor impairment observed in D2R-null mice 4 does not adversely affect the motor responses elicited either by exogenous or endogenous opioids. Thus the mechanisms responsible for opioid-induced enhancement of locomotion are not altered in D2R-null mice, in agreement with the previously proposed participation of $\mathrm{D} 1 \mathrm{Rs}^{17}$ and of non-dopaminergic mechanisms ${ }^{19,20}$ in this response.

To investigate whether the reward properties elicited by morphine administration were still observable in D2R-deficient mice, wildtype and D2R-null morphine-treated mice ( $n=8$ per group) were analysed by using the place-conditioning paradigm ${ }^{21}$. During the

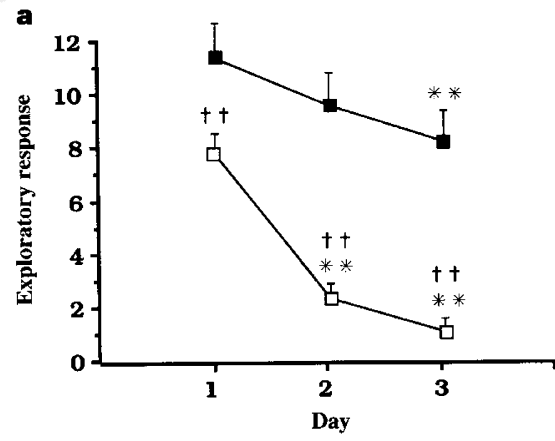

Figure 1 D2R-null mice preserve exploratory activity and locomotor response to morphine treatment. a, Exploratory response of D2R-null mice (open squares), and wild-type littermates (filled squares) in a stressful (highly illuminated) open field containing unknown objects. This test was performed for three consecutive days, at 24-h intervals; values represent the number of visits to the unknown objects (mean \pm s.e.m.). The number of squares crossed (mean \pm s.e.m.) for the wild-type mice were: day $1,228.7 \pm 10.01 ; 2,194.8 \pm 8.2 ; 3,161 \pm 11.9 ; \mathrm{D} 2 \mathrm{R}$ mutants, day $1,154 \pm 16.6 ; 2,61 \pm 8.4 ; 3,47.4 \pm 4$. Number of rearings for wild-

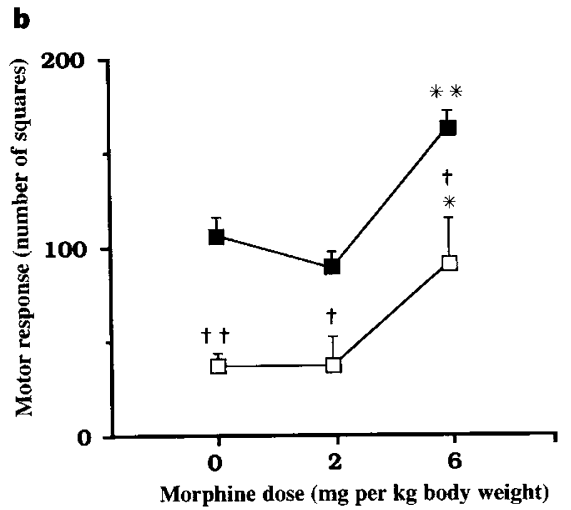

type day $1,30 \pm 1.5 ; 2,20.8 \pm 2.1 ; 3,18.8 \pm 1.8$; for D2R mutants, day 1, $6.1 \pm 1.2 ; 2$, $1.9 \pm 0.6,3,1.2 \pm 0.4$. ${ }^{*} P$ versus same group on day 1 (Dunnett's test); $\dagger \dagger P<0.01$ versus wild-type group (Student's $t$-test); $n=12$ per group. $\mathbf{b}$, Motor responses to morphine administration (mean \pm s.e.m.) in D2R-null (open squares) and wildtype (filled squares) mice, in a non-stressful environment. ${ }^{\star} P<0.05,{ }^{*} P<0.01$, versus saline group with the same genotype; $\uparrow P<0.05$, $\uparrow \uparrow P<0.01$, versus wildtype group receiving the same treatment (Dunnett's test); $n=6$ per group. 
preconditioning phase, D2R-null and wild-type mice both visited the two compartments of the place-preference apparatus (Table 1), spending the same time in each compartment (data not shown). Two other groups of animals with the same genotypes were administered saline as control. Morphine administration (3 mg perkg body weight, subcutaneous (s.c.)) induced a clear conditioned place preference in wild-type mice, indicated by a significant increase in the time spent in the drug-associated compartment during the testing phase. This conditioned behaviour is completely absent in D2R mutant mice, which spent the same time in the morphine-designed compartment during the preconditioning and the testing phases (Fig. 2a, b). To exclude the possibility that tolerance might have arisen in D2R-null mice as a result of an endogenous higher level of enkephalins ${ }^{4}$, the same experiment was repeated in a new group of drug-naive animals using a higher dose ( $9 \mathrm{mg}$ per $\mathrm{kg}$ body weight s.c.) of morphine ( $n=8$ per group). In these conditions, D2R-null mice, in contrast to wild-type animals, also failed to show place preference (Fig. 2a,b). Thus the lack of place preference is not due to a developed tolerance to endogenous opioids. Motor and motivational responses to opioids have been reported to be closely related ${ }^{22,23}$. However, absence of place preference is not dependent on the locomotor impairment of D2R-null mice, as these mice preserve a motor response to endogenous (RB 101 administration) and exogenous opioids (Fig. 1b); show a behaviour similar to that of wild-type mice during the preconditioning phase, including the number of visits to each compartment (Table 1); and maintain the locomotor and exploratory potential to respond to the environment (Fig. 1). Thus there is a dissociation between opioid-mediated motor and motivational responses.

The ability of D2R-null mice to show preference to other incentive stimuli was also investigated, by evaluating their condi- tioned response to palatable food in the same place-preference paradigm. Food reward produced the same conditioned place preference in both wild-type $(n=10)$ and D2R-null mice $(n=10)$, as indicated by a significant increase in the time spent in the food-associated compartment during the testing phase (Fig. $2 \mathrm{c}, \mathrm{d})$. No place preference was observed in the control groups. Therefore the motivational deficit produced by the absence of D2Rs selectively affects morphine rewarding properties, but does not modify food-induced place preference. This suggests that there is a specific involvement of D2R in the motivational component of opioid dependence, but not on natural rewards.

It is known that $\mu$-opioid receptors are responsible for morphineinduced place conditioned preference ${ }^{24}$. We investigated whether changes in the expression of this receptor in the D2R-null background might be responsible for loss of morphine reward. Analysis of striatal and ventral mesencephalic mRNA and proteins from wild-type and D2R-null mice showed there to be a similar level of expression of this gene and of receptor binding in both genotypes (Fig. 3, and data not shown). Consequently, lack of place preference in D2R-null mice is dependent on the loss of the rewarding properties of morphine resulting from the absence of dopamine D2Rs.

We then investigated the participation of D2R in the physical dependence on opiates. The activation of $\mathrm{D} 2 \mathrm{R}$ within the nucleus accumbens has been reported to reduce the severity of the naloxoneprecipitated morphine-withdrawal syndrome, whereas its blockade in morphine-dependent animals precipitates abstinence ${ }^{2}$. A severe degree of dependence was induced in wild-type and D2R-null mice by chronic administration of increasing doses of morphine. Control animals of the same genotypes were treated with saline $(n=8$ per group). The manifestation of the somatic symptoms of withdrawal was evaluated after administration of naloxone. Chronic morphine
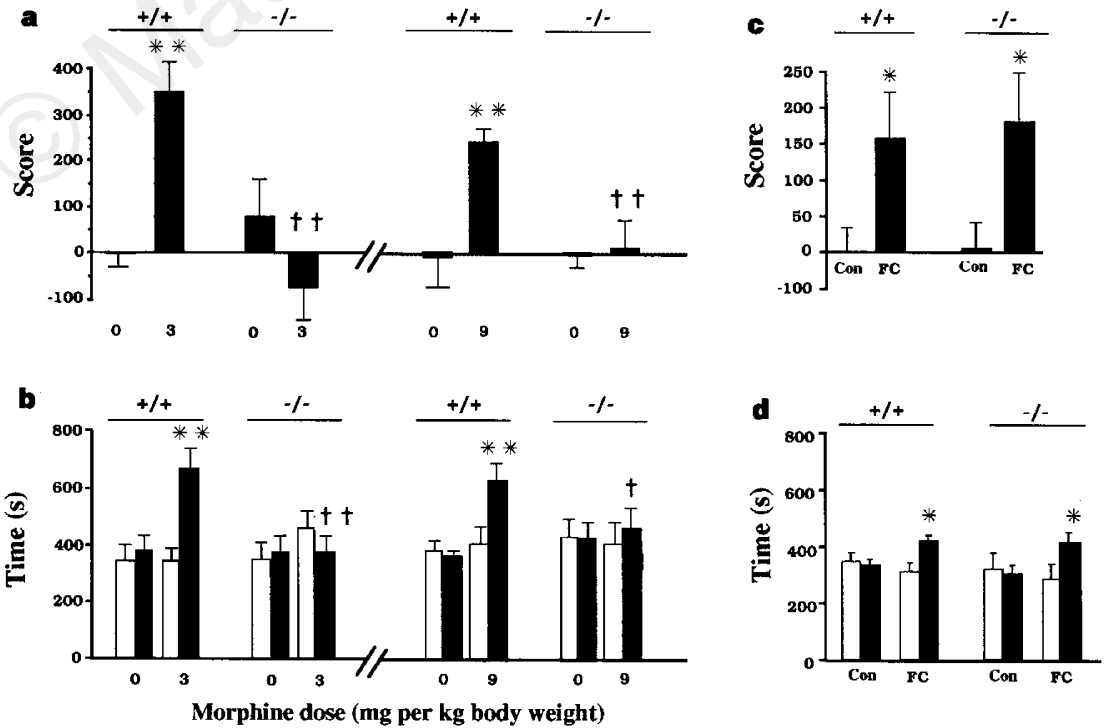

Figure 2 Lack of morphine-induced place preference in D2R-null mice. a, b Conditioned place preference induced by morphine in D2R-null mice and wildtype littermates. a, Score ${ }^{21}$ calculated as the difference between postconditioning and preconditioning time spent in the compartment associated with morphine. b. Time spent in the drug-associated compartment during the preconditioning (white bars) and the testing phases (black bars). (Values represent mean \pm s.e.m.). ${ }^{* *} P<0.01$, versus saline group with the same genotype $\dagger P<0.05$, $\uparrow \dagger P<0.01$, versus wild-type group receiving the same treatment (Dunnett's test); $n=8$ per group. The comparison within each group of the time spent during the preconditioning and testing phases in the morphine-associated compartment (Student's $t$-test) revealed a significant difference $(P<0.01)$ in wildtype mice treated with 3 and $9 \mathrm{mg}$ per $\mathrm{kg}$ morphine (data not shown). c, d,
Conditioned place preference induced by food reward in D2R-null mice and their wild-type littermates. c, Scores (Con, control group; FC, food-conditioned group) calculated as the difference between postconditioning and preconditioning time spent in the compartment associated with food. d, Time spent in the foodassociated compartment during the preconditioning (white bars) and testing (black bars) phases. ${ }^{*} P<0.05$, versus the non-conditioned group with the same genotype (Dunnett's test); $n=10$ per group. The comparison within each group of the time spent during the preconditioning and testing phases in the foodassociated compartment (Student's $t$-test) also revealed a significant difference $(P<0.05)$ in food-conditioned wild-type D2R-null mice (not shown). Genotypes are indicated above the columns. 


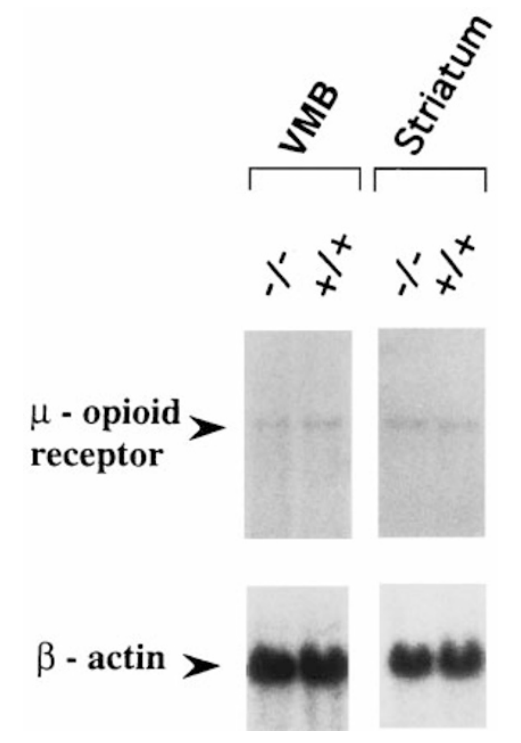

Figure $3 \mu$-Opioid receptor expression in D2R-null mice. Northern blot analysis of $\mu$-opioid receptor mRNA expression in striatum and ventral midbrain (VMB) of wild type $(+/+)$ and homozygous (-/-) D2R-null mice ( $n=3$ per group). $\beta$-Actin was used as an internal control. Bands corresponding to the $\mu$-receptor and $\beta$ actin mRNAs are indicated.

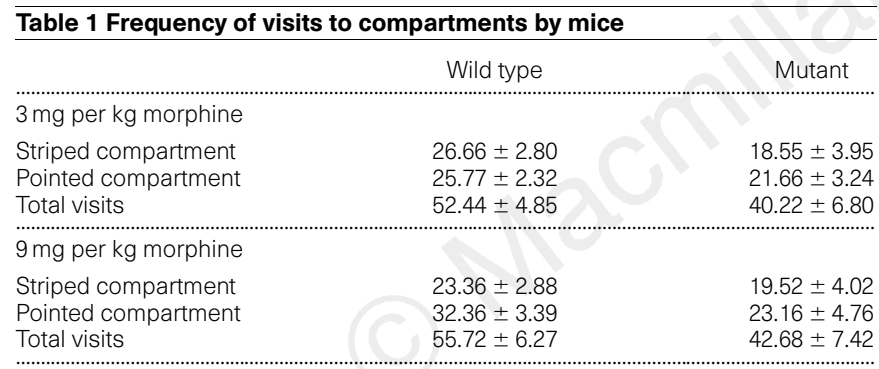

Results (mean \pm s.e.m.) are for visits during the preconditioning phase.

treatment induced the classical signs of opiate exposure, such as Straub reflex and increased locomotor activity in both groups of animals (data not shown). Naloxone administration had no effect on the saline-injected groups; conversely, it strongly precipitated the behavioural symptoms of withdrawal in morphine-treated animals. No differences between D2R-null and wild-type mice were observed (Fig. 4), apart from sniffing incidence, which was significantly higher in D2R mutant mice. However, sniffing is a minor sign of abstinence and has little significance in the evaluation of withdrawal severity. Indeed, the global withdrawal scores, calculated for each animal by using a range of possible scores ${ }^{25}$ from 0 to 100 , were similar in mutant $(64 \pm 6)$ and wild-type mice $(58 \pm 5)$.

These data indicate that although D2 receptors in the nucleus accumbens might be involved in the modulation of physical opiate dependence 2 , they are not required to obtain a complete manifestation of the somatic signs of opiate withdrawal. In agreement with this finding, local administration of an opiate antagonist into the nucleus accumbens did not induce any behavioural sign of withdrawal in morphine-dependent rats ${ }^{15}$. Morphine infusion into this area has also failed to produce an important degree of physical dependence ${ }^{16}$.

Previous studies of the involvement of the dopaminergic system in opiate rewarding effects have led to conflicting results ${ }^{8,12-14,26-29}$. The discrepancies between these reports are probably due to the experimental conditions used, the pharmacological specificity of
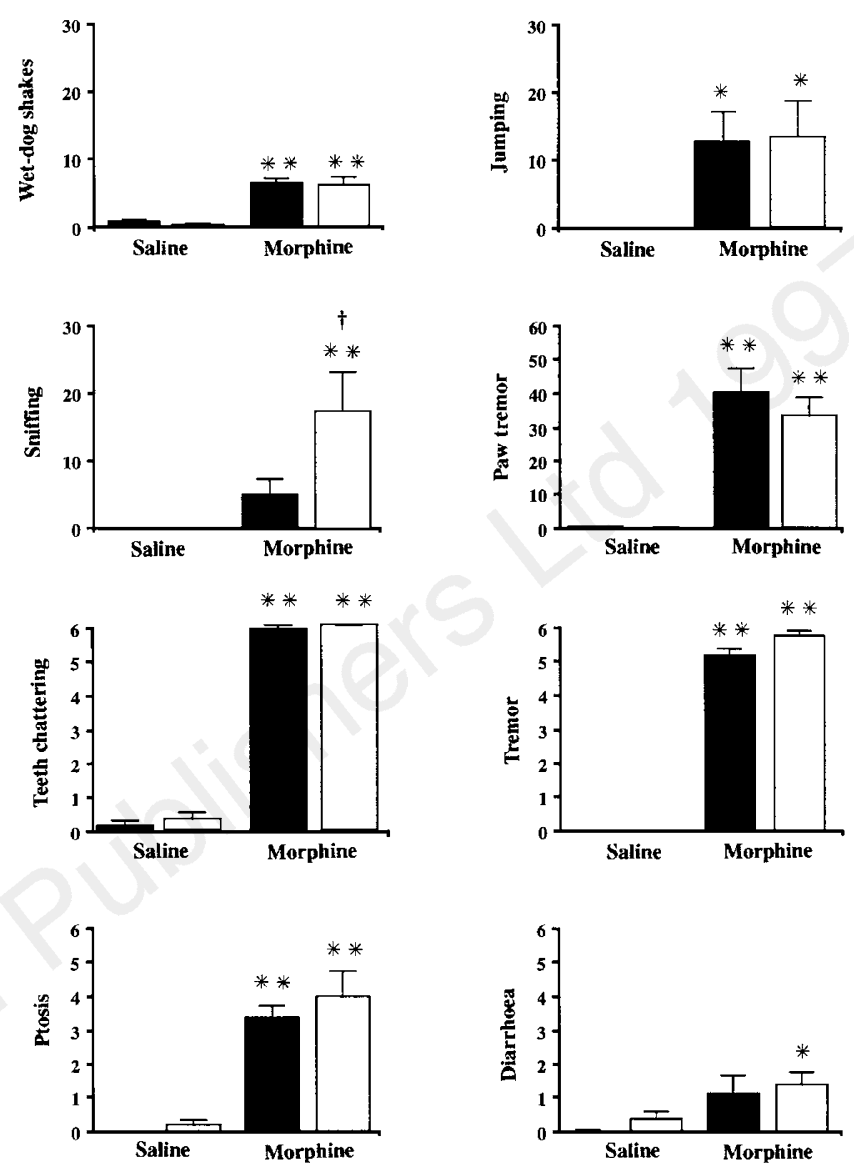

Figure 4 Analysis of morphine-induced physical dependence. Incidence of somatic signs of abstinence (mean \pm s.e.m.) measured during naloxone-precipitated morphine-withdrawal syndrome in D2R-null mice (white bars) and wild-type littermates (black bars). ${ }^{\star} P<0.05,{ }^{*} P<0.01$, versus saline group with the same genotype; $\uparrow P<0.05$, versus wild-type group receiving the same treatment (Dunnett's test); $n=8$ per group.

the D2 antagonists, and/or the dose ranges studied. The use of D2Rnull mice as a model system allows the use of intact animals in which the absence of D2R expression is obtained by genetic manipulation. Our results have unambiguously demonstrated a dissociation between the mechanisms involved in the rewarding properties of opiates, which are related to their addictive capacities, and the somatic signs of the naloxone-precipitated withdrawal syndrome, which reveal the physical component of opiate dependence. Thus dopaminergic D2 receptors are crucial for the rewarding effects of opiates, but not for the development of physical opiate dependence or the expression of some acute responses to morphine, such as hypermotility. We have also demonstrated a selective physiological involvement of D2Rs in some forms of drug-mediated reward that is different from that produced using other rewarding stimuli.

\section{Methods}

The D2R (-/-) mice and wild-type (+/+) littermates were from the mating of heterozygous D2R-null mice ${ }^{4}$. Morphine- $\mathrm{HCl}$ and naloxone- $\mathrm{HCl}$ were from Sigma, and RB101 was produced as described ${ }^{18}$. All behavioural and pharmacological experiments were performed in a soundproof room by an observer who did not know either the genotype of the mice or the pharmacological treatment.

Open field test. For stressful conditions, a large white rectangular box was used $(70-\mathrm{cm}$ wide, $90-\mathrm{cm}$ long and $60-\mathrm{cm}$ high) that was brightly illuminated from the top (500 lux) to present four unknown objects, placed $20 \mathrm{~cm}$ from 
each corner. A total of 63 squares $(10 \times 10 \mathrm{~cm})$ were drawn with black lines on the white floor of the field. The presence of six events was quantified during an observation period of $5 \mathrm{~min}$ : squares crossed, rearings, grooming bouts, defecation boli, urination events and visits to the unknown objects. Values were analysed by two-way analysis of variance (ANOVA) with repeated measures (time, within subjects; genotype, between subjects). After main effect of ANOVA, individual comparisons within subjects were made by the two-tailed Dunnett's test, and between subjects by the two-tailed Student's $t$ test.

Locomotor activity boxes. In non-stressful conditions, locomotion was evaluated in locomotor activity boxes $10 \mathrm{~min}$ after saline or morphine- $\mathrm{HCl}(2$ and $6 \mathrm{mg}$ per $\mathrm{kg}$ body weight, s.c.) administration in a transparent rectangular box $(30 \times 26 \times 30 \mathrm{~cm})$ with a metallic grid floor and moderate illumination (50 lux). Mice were not habituated to the test box. Animal displacements were measured by drawing 12 squares on the floor. The number of squares crossed and the number of rearings were counted during a period of $10 \mathrm{~min}$. Values were analysed by two-way ANOVA (genotype and treatment) between subjects. After main effect of ANOVA, individual comparisons between groups were made by two-tailed Dunnett's test.

Place-preference paradigm. Tests were performed as previously reported ${ }^{21}$, except for the conditioning time $(18 \mathrm{~min})$ and the size of compartments $(15 \times 15 \times 15 \mathrm{~cm})$. One compartment of the place-preference apparatus is randomly chosen to be paired to drug administration and the other to saline. The drug-assigned compartment could be either the most or the least preferred. Care was taken to ensure that treatments were counterbalanced as closely as possible between compartments. Experiments were conducted during the light phase of the dark/light cycle. The place-preference conditioning schedule consisted of three phases. (1) Preconditioning phase, during which mice were placed in the middle of the neutral area and their location was recorded for the $18 \mathrm{~min}$. After the session, animals were randomized to be paired to drug or vehicle administration and to be assigned to a compartment. (2) Conditioning phase, during which mice were treated for 6 consecutive days with alternate injections of morphine- $\mathrm{HCl}$ ( 3 or $9 \mathrm{mg}$ per $\mathrm{kg}$ body weight, s.c.) or saline. Doors matching the walls of the compartment allowed the confinement of the mice for $20 \mathrm{~min}$ immediately after morphine or saline injections. Mice received the drug on days 1, 3 and 5, and vehicle on days 2, 4 and 6. Control animals received vehicle every day. (3) Testing phase, which was conducted exactly as the preconditioning phase (free access to each compartment for $18 \mathrm{~min}, 24 \mathrm{~h}$ after the final conditioning session). Food reward was tested in animals with limited access to food (normal mouse food plus sucrose) for 5 days before the test. The same procedure was used as for morphine, with the exception that mice conditioned to food had free access to it (normal mouse food plus sucrose) in the confined compartment on days 1, 3 and 5, and had no access to food in the other compartment on days 2, 4 and 6. Control animals had no access to food in the assigned compartments during this phase (days 16). A fixed amount of food equivalent to $12 \%$ of mouse body weight was applied per day, as previously reported ${ }^{30}$. Animals were maintained on the same diet throughout the experimental period. Data were analysed by two-way ANOVA (genotype and treatment) between subjects. Individual comparisons were made in each case by the two-tailed Dunnett's test, after main effect of ANOVA.

Analysis of abstinence somatic signs. Opiate dependence was induced in mice by repeated i.p. injection of morphine- $\mathrm{HCl}$, at an interval of $12 \mathrm{~h}$, for 6 days. The morphine- $\mathrm{HCl}$ dose was progressively increased as follows: day 1 , $10 \mathrm{mg}$ per kg body weight; 2, $20 \mathrm{mg}$ per kg; 3, $30 \mathrm{mg}$ per kg; 4, $40 \mathrm{mg}$ per kg; 5, $50 \mathrm{mg}$ per kg; 6 (only one injection in the morning), $50 \mathrm{mg}$ per kg. Control mice were treated with saline under the same conditions. Withdrawal was precipitated only once in each animal by injecting naloxone- $\mathrm{HCl}(1 \mathrm{mg}$ per $\mathrm{kg}$, s.c.) $2 \mathrm{~h}$ after the last morphine- $\mathrm{HCl}$ administration. Mice were placed individually into test chambers consisting of transparent round plastic boxes ( $30 \mathrm{~cm}$ diameter, $50 \mathrm{~cm}$ height) with a white floor $30 \mathrm{~min}$ before naloxone- $\mathrm{HCl}$ injection. During the $15 \mathrm{~min}$ before naloxone- $\mathrm{HCl}$ injection, mice were observed to verify the presence of normal behaviour. Somatic signs of withdrawal were evaluated immediately after the injection of the opiate antagonist during a period of $30 \mathrm{~min}$. The number of wet-dog shakes, jumping, sniffing and paw tremor were counted. Teeth chattering, diarrhoea, tremor and ptosis were evaluated over periods of $5 \mathrm{~min}$, one point being given for the presence of each sign during each period. The number of periods showing the sign was then counted (maximum score of 6 ). Values were analysed by two-way ANOVA (genotype and treatment) between subjects. Individual comparisons were made by the two-tailed Dunnett's test, after main effect of ANOVA.

Northern blot analysis. Total RNA from striatum and ventral midbrain was purified by the lithium chloride method, and $10 \mu \mathrm{g}$ RNA was analysed by northern blot. The ${ }^{32} \mathrm{P}$ random primed probe used to reveal the $\mu$-opioid receptor mRNA corresponds to the 870 base-pair fragment spanning base pairs 785 to 1653 of the mouse $\mu$-opioid receptor cDNA ${ }^{24}$.

Received 4 November 1996; accepted 9 May 1997.

1. Wise, R. A. \& Bozarth, M. A. A psychomotor stimulant theory of addiction. Psychol. Rev. 97, 469-492 (1987)

2. Harris, G. C. \& Aston-Jones, G. Involvement of D2 dopamine receptors in the nucleus accumbens in the opiate withdrawal syndrome. Nature 371, 155-157 (1994).

3. Koob, G. F. Drugs of abuse: anatomy, pharmacology and function of reward pathways. Trends Pharmacol. Sci. 13, 177-184 (1992).

4. Baik, J. H. et al. Parkinsonian-like locomotor impairment in mice lacking dopamine D2 receptors. Nature 377, 424-428 (1995).

5. Di Chiara, G. \& Imperato, A. Drugs abused by humans preferentially increase synaptic dopamine concentrations in the mesolimbic system of freely moving rats. Proc. Natl Acad. Sci. USA 85, 52745278 (1988).

6. Wise, R. A. Neurobiology of addiction. Curr. Opin. Neurobiol. 6, 243-251 (1996).

7. Bozarth, M. A. \& Wise, R. A. Intracranial self-adminsitration of morphine into the ventral tegmental area in rats. Life Sci. 28, 551-555 (1981).

8. Broekkamp, C. L., Phillips, A. S. \& Cools, A. R. Facilitation of self-stimulation behavior following intracerebral microinjections of opioids into the ventral tegmental area. Pharmacol. Biochem. Behav. 11, 289-295 (1979).

9. Phillips, A. C. \& Le Piane, E. G. Reinforcing effects of morphine microinjection into the ventral tegmental area. Pharmacol. Biochem. Behav. 12, 965-968 (1980).

10. Joyce, E. M. \& Iversen, S. D. The effect of morphine applied locally to mesencephalic dopamine cell bodies on spontaneous motor activity in the rat. Neurosci. Lett. 14, 207-212 (1979).

11. Kalivas, P. W. \& Richardson-Carlson, R. Endogenous enkephalin modulation of dopamine neurons in ventral tegmental area. Am. J. Physiol. 251, R243-R249 (1986).

12. Ettenberg, A., Pettit, H. O., Bloom, F. E. \& Koob, G. F. Heroin and cocaine intravenous selfadministration in rats: mediation by separate neural systems. Psychopharmacology 78, 204-209 (1982).

13. Pettit, H. O., Ettenberg, A., Bloom, F. E. \& Koob, G. F. Destruction of dopamine in the nucleus accumbens selectively attenuates cocaine but not heroin self-administration in rats. Psychopharmacology 84, 167-173 (1984).

14. Borg, P. J. \& Taylor, D. A. Voluntary oral morphine self-administration in rats: effect of haloperidol or ondansetron. Pharmacol. Biochem. Behav. 47, 633-646 (1994).

15. Maldonado, R., Stinus, L., Gold, L. \& Koob, G. F. Role of different brain structures in the expression of the physical morphine withdrawal syndrome. J. Pharmacol. Exp. Ther. 261, 669-677 (1992).

16. Bozarth, M. A. Physical dependence produced by central morphine infusions: an anatomical mapping study. Neurosci. Biobehav. Rev. 18, 373-383 (1994).

17. Longoni, R., Spina, L. \& Di Chiara. Dopaminergic D-1 receptors: essential role in morphine-induced hypermotility. Psychopharmacology 93, 401-402 (1987)

18. Fournié-Zaluski, M. C. et al. "Mixed inhibitor-prodrug" as a new approach toward systemically active inhibitors of enkephalin-degrading enzymes. J. Med. Chem. 35, 2473-2481 (1992).

19. Kalivas, P. W., Widerlow, E., Stanley, D., Breese, G. \& Prange, A. J. Enkephalin action on the mesolimbic system: a dopamine-dependent and a dopamine-independent increase in locomotor activity. J. Pharmacol. Exp. Ther. 227, 229-237 (1983).

20. Daugé, V., Rossignol, P. \& Roques, B. P. Blockade of dopamine receptors reverses the behavioral effects of edogenous enkephalins in the nucleus caudatus but not in the nucleus accumbens: differential involvement of delta and mu opioid receptors. Psychopharmacology 99, 168-175 (1989).

21. Valverde, O., Fournié-Zaluski, M. C., Roques, B. P. \& Maldonado, R. The CCKв antagonist PD134,308 facilitates rewarding effects of endogenous enkephalins but does not induce place preference in rats. Psychopharmacology 123, 119-126 (1995).

22. Salamone, J. D. The involvement of nucleus accumbens dopamine in appetitive and aversive motivation. Behav. Brain. Res. 61, 117-133 (1994).

23. Salamone, J. D. The behavioral neurochemistry of motivation: methodological and conceptual issues in studies of the dynamic activity of nucleus accumbens dopamine. J. Neurosci. Methods 64, 137-149 (1996).

24. Matthes, H. W. D. et al. Loss of morphine-induced analgesia, reward effect and withdrawal symptoms in mice lacking the $\mu$-opioid-receptor gene. Nature 383, 819-823 (1996).

25. Maldonado, R., Negus, S. \& Koob, G. F. Precipitation of morphine withdrawal syndrome in rats by administraiton of mu-, delta- and kappa-selective opioid antagonists. Neuropharmacology 31, 12311241 (1992).

26. Spyraki, C., Fibiger, H. C. \& Phillips, A. G. Attenuation of heroin reward in rats by disruption of the mesolimbic dopamine system. Psychopharmacology 79, 278-283 (1983).

27. Mackey, W. B. \& van der Kooy, D. Neuroleptics block the positive reinforcing effects of amphetamine but not of morphine as measured by place conditioning. Pharmacol. Biochem. Behav. 22, 101-105 (1985).

28. Stinus, L. et al. Chronic flupentixol treatment potentiates the reinforcing properties of systemic heroin administration. Biol. Psychiat. 26, 363-371 (1989)

29. Smith, J. E., Guerin, G. F., Co, C., Barr, T. S. \& Lane, J. D. Effects of 6-OHDA lesions of the central medial nucleus accumbens on rat intravenous morphine self-administration. Pharmacol. Biochem. Behav. 23, 843-849 (1985).

30. Elmer, G. I., Pieper, J. O., Goldberg, S. R. \& George F. R. Opioid operant self-administration, analgesia, stimulation and respiratory depression in $\mu$-deficient mice. Psychopharmacology 117, 23-31 (1995).

Acknowledgements. We acknowledge B. Kieffer for the gift of $\mu$-opioid cDNA. A.S. and O.V. were recipient of fellowships from the European Community and T.A.S. from the Centre Nationale de la Recherche Scientifique (CNRS). This work was supported by funds from the Ministère de la Recherche to R.M., from the Association pour la Recherche sur le Cancer to R.M. and to E.B., and from the Institut Nationale de la Santé et de la Recherche Médicale, CNRS et Centre Hospitalier Universitaire Regional to E.B.

Correspondence and requests for materials should be addressed to E.B. (e-mail: eb@titus.u-strasb.fr). 